\title{
Paraquat intoxication as a cause of multiple organ failure: report of a case and review of the literature
}

\begin{abstract}
Introduction: Paraquat is the most commonly used herbicide in the world, due to its hydrophilic characteristics it is easily absorbed by mucous membranes, presenting a high affinity for several organs, mainly the lung. The mechanisms of direct injury given by this toxic are: the generation of free radicals (paraquat + ), reactive oxygen species and direct mitochondrial lesion, which subsequently cause the release of a systemic inflammatory response and end in multiple organ failure, in this condition circulating cells, endothelial cells and different inflammatory mediators interact. Case presentation: We present the case of a 34-year-old male patient with a diagnosis of paraquat intoxication that evolved to multiple organic failure and death in the short term.
\end{abstract}

Discussion: In the present case we note the failure of three organs on admission to the emergency room, with an APACHE II score of 18 points, SOFA of 11 points, SAPS II of points and MODS 74 points, demonstrating a mortality that ranges between 30 and 88 $\%$ basically due to the exaggerated abnormal inflammatory response over the entire body sistems. Although the patient was managed with an organic ventilatory and renal support in a protective manner, the toxicity generated an irreversible systemic damage that ended in the death of the patient.

Conclusion: Paraquat increases its pulmonary concentration during the first hours postintake due to its high affinity to alveolar cells. Due to this, injury to the pneumocytes occurs due to the reduction of paraquat to free radical cations (paraquat + ), a process initiated by the NADH. The reactive oxygen species cause a systemic inflammatory response, based on cytokines and cellular infiltration that finally conclude in the peroxidation of the cellular lipid membrane, which in general terms is the basis of generation of multiple organ failure mainly in lung, kidney and liver. The mitochondrial lesion releases iron-free forms, generating greater production of free radicals and systemic apoptosis and necrosis.

Keywords: paraquat, multiple organ failure, mitochondrial lesion, free radicals, systemic inflammatory response

\author{
Volume 4 Issue 4 - 2018
}

\author{
Miño Bernal Jorge Fernando,' Martínez \\ Ortega Juan Camilo, ${ }^{2}$ Pérez Nieto Orlando, ${ }^{3}$ \\ Romo Caicedo Jesús ${ }^{2}$ \\ 'Emergency Physician,Attached Unit of Intensive Care, \\ Colombia \\ ${ }^{2}$ Emergency Service, Las Lajas Clinic, Colombia \\ ${ }^{3}$ Intensive Care Unit, San Juan del Río General Hospital, Mexico
}

Correspondence: Orlando Rubén Pérez Nieto, Intensive Care Unit, Hospital General San Juan del Río, Queretaro, Boulevard Luis Donaldo Colosio 422, col. Sagrado corazón, San Juan del Río, Querétaro, Tel 01-52 5510154428, Mexico,

Email orlando_rpn@hotmail.com

Received: June 28, 2018 | Published: July II, 2018

\section{Introduction}

Paraquat is the most commonly used herbicide in the world, due to its hydrophilic characteristics it is easily absorbed by mucous membranes, presenting a high affinity for several organs, mainly the lung. The mechanisms of direct injury given by this toxic are: the generation of free radicals (paraquat + ), reactive oxygen species and direct mitochondrial lesion, which subsequently cause the release of a systemic inflammatory response and end in multiple organ failure, in this condition circulating cells, endothelial cells and different inflammatory mediators interact.

\section{Case presentation}

We present the case of a 34-year-old male patient, referred from the Tumaco Hospital with a diagnosis of Gramoxone intoxication, brought on a stretcher by his family member who states that the clinical features started in the previous 30hours, consisting of voluntary intake of pesticide (gramoxone+paraquat), with persistent ideation and suicidal plan, associated with an episode of abundant vomiting caused by relatives after administration of water with salt. The initial management in the Tumaco Hospital was with intravenous fluids and urinary Foley catheter placement, gastric lavage was not performed since the patient does not cooperate for the procedures. The patient does not present a significant pathological history. The vital signs of admission are: CR 110 ', RR 34', AP 112/54 mmHg, Temp $36.7^{\circ} \mathrm{C}, \mathrm{SaO}_{2}: 80 \%$. The physical examination is the following:
The patient was found to have a 6-point Glasgow coma scale, with isochoric pupils, reactive to the light, icteric scleras, oral wet mucosa with tongue and mucosal defacement, movable neck, not painful, without signs of meningeal irritation, you do not feel any adenoids or masses (Figure 1) symmetrical thorax, normoexpansible, rhythmic heart sounds, no murmurs, vesicular murmur present in both lung fields, bilateral bibasal crepitant rales are auscultated, the abdomen is soft, depressable, painful on palpation in the epigastrium, without signs of peritoneal irritation, no masses or megalia are palpated, normal peristalsis, the extremities are eutrophic, mobile, positive distal pulses, capillary filling less than two seconds, no edema.

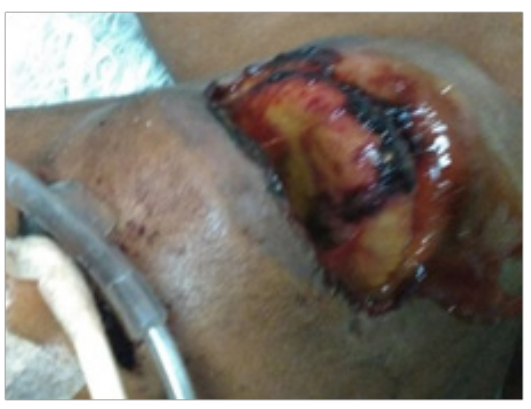

Figure I Photograph of the patient of the described case, the oral mucosa is observed with edema, jaundice and ulcerations. 
In laboratory studies, hypoxia is reported due to increased lactate plus hypoxemia due to a decrease in partial blood pressure of oxygen, severe acute respiratory distress syndrome, leukocytosis with neutrophilia, elevated C-reactive protein, hyponatremia, hypocalcemia, and urea nitrogen. High creatinine, calculated glomerular filtration rate of $15.6 \mathrm{cckg} / \mathrm{min} / \mathrm{m} 2$ and a BUN / Cr ratio of 18.4; Hyperbilirubinemia is found at the expense of direct bilirubin, increased transaminase levels, normal coagulation tests, and hyperamylasemia. The chest radiograph shows bilateral opacities of peripheral and parahilar predominance (Figure 2). Respiratory failure is worthy of or tracheal intubation and application of mechanical protective ventilation, intravenous fluid administration continues, intravenous steroid is initiated, enteral $\mathrm{N}$-acetylcysteine, Vitamin E, Vitamin A, Vitamin C, intravenous Colchicine, loop diuretic, thromboprophylaxis and prophylaxis of stress ulcers. He is admitted to the intensive care unit where renal replacement therapy begins.

The patient's diagnoses are the following:

a) Intoxication by herbicide-type pesticides

b) Paraquat intoxication

c) SADPERSON suicide attempt score: 6 points

d) Multiple Organic Failure

e) Hypoxemic respiratory insufficiency

f) Acute respiratory distress syndrome

g) Hypoxia

h) Hyperacute fulminating hepatic failure

i) Acute kidney injury AKI III

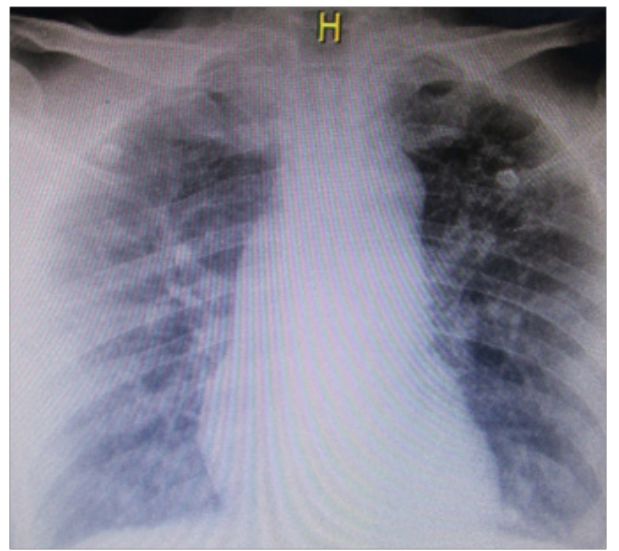

Figure 2 Chest radiograph of the patient of the described case, that shows bilateral opacities of peripheral and parahilar predominance.

After 10 days of hospitalization in the intensive care unit with mechanical protective ventilation, renal replacement therapy, treatment of liver failure, glycemic control and intensive care, patients die, results of necrosis are obtained, in which the following are reported : liver enlarged in size and weight, congestive and indurated, gallbladder with cholestasis (Figure 3), lungs increased in consistency and weight, hard to the touch of violaceous color, with whitish areas, with speckled appearance with alternating dark areas with another yellowish tint lighter, generalized and fibrosis (Figure 4), edematous kidneys, with smooth brown surface, adequate medullary cortical configuration, yellowish tone in generalized form Figure 5.

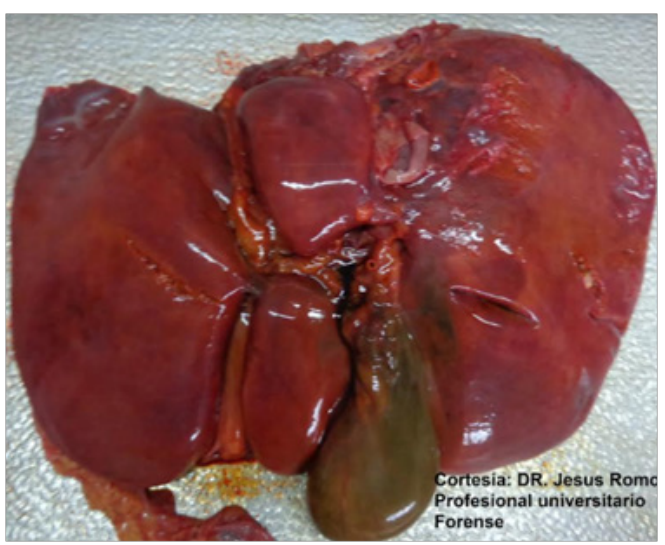

Figure 3 Liver enlarged in size and weight, congestive and indurated, gallbladder with cholestasis. Photograph taken from the pathology study of the patient of the described case, courtesy of the forensic doctor Jesús Romo Caicedo.

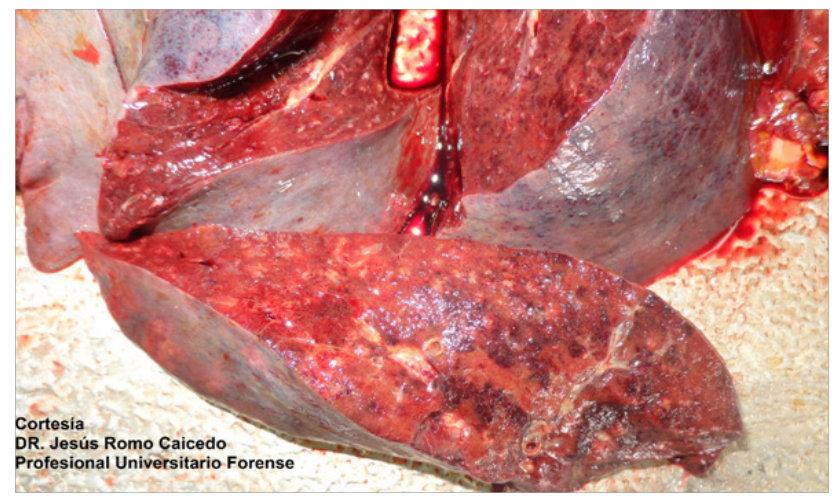

Figure 4 Lungs increased in consistency and weight, hard to the touch of violaceous color, with whitish areas, with mottled appearance with alternation of dark areas with another lighter, generalized yellowish tint and fibrosis. Photograph taken from the pathology study of the patient of the described case, courtesy of the forensic doctor Jesús Romo Caicedo.

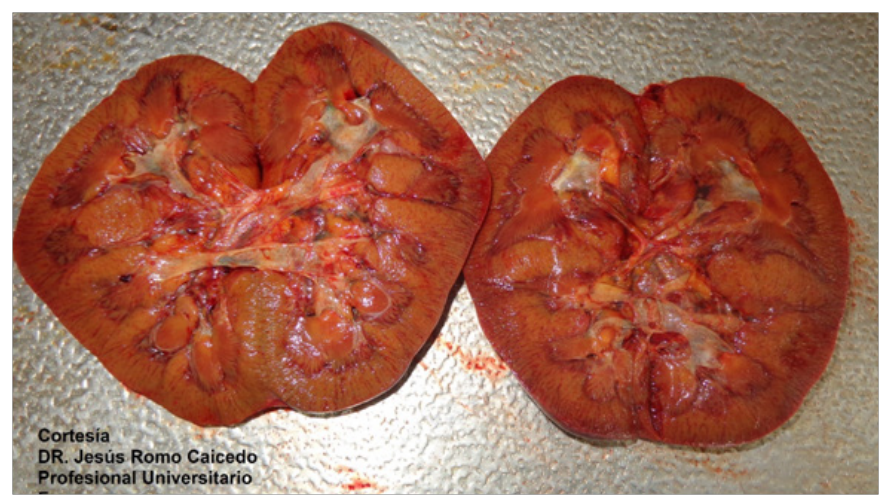

Figure 5 Edematous, symmetric kidneys with smooth brown surface, adequate medullary cortical configuration, yellowish tint in generalized form. Photograph taken from the pathology study of the patient of the described case, courtesy of the forensic doctor Jesús Romo Caicedo.

\section{Review of literature}

Paraquat (N, N'-dimethyl-4, 4'-dichlorobipridinium) is one of the most widely used herbicides in the world, and although it is banned in the European Union, it is still used in developing countries. There are 
reports in Asian countries where they report an annual incidence of 2000 intakes with a mortality of $60 \% \cdot{ }^{1-3}$ It is a non-selective herbicide, inactivated in the soil and adheres strongly to clays. It is a watersoluble compound that is easily ionized, stable in acid solutions and unstable in alkaline solutions. Due to its hydrophilic characteristics its main absorption route is the oral route, its persistence in the circulatory tissue is low. It accumulates in some tissues and its largest white organ is the lung from there. ${ }^{1-3}$

\section{Mechanisms of cytociicity}

\section{Formation of the radicals of Paraquat ${ }^{4}$}

Theory of origin of the microsomal fraction: The mechanism of paraquat cytotoxicity was first explained by the formation of paraquat radicals and viamicrosomal lipid peroxidation of NADPH-cytochrome $\mathrm{C}$ reductase. Paraquat is reduced to NADH by cytochrome $\mathrm{C}$ reductase, and, reduced paraquat is reoxidized by cytochrome $\mathrm{P}-450$, in the presence of tertiary amines. The interaction of paraquat with the NADH cytochrome P-450 reductase and ferric complexes results in an increase in the generation of free radicals. Paraquat stimulates the production of hydrogen peroxide, superoxide radicals and hydroxyl radicals.

Theory of microsomal origin: It proposes that paraquat affects mainly at the level of the ultrastructure of the alveolar cells of type II pneumocytes, with the mitochondria being the objective organelle (the main site for the formation of radicals).

A. Oxidareductase NADH-quinone from the mitochondrial outer membrane: paraquat is anaerobically reduced by the intact mitochondria in the presence of NADH. Oxygen radicals are produced during the oxidation of NADH in the mitochondrial outer membrane.

B. Oxidareductase NADH-quinone from the inner mitochondrial membrane: Paraquat has been shown to decouple mitochondrial oxidative phosphorylation, stimulating basal oxygen consumption without using phosphorylation of adenosindiphosphate (ADP).

The toxic mechanisms and metabolic effects of paraquat were observed in samples of renal tubules of exposed rabbits, where high concentrations of paraquat altered the chain of mitochondrial electron transfer, thus reducing the metabolic functions of the affected tissues. On the other hand, paraquat induces lipid peroxidation dependent on NADH.

\section{The role of paraquat-induced free radical formation in cell injury ${ }^{4}$}

There is a balance between oxidizing agents that damage different tissues and their neutralization with antioxidants. Reactive oxygen species are involved in the formation of ferric and nitrogenous species that act on direct cell destruction, and as secondary messengers in the inflammatory cascade. The balance between reactive oxygen species and nitrogenous reactive species determines the progression to multiple organ failure, this is exacerbated by the production of paraquat free radicals as agents of direct injury, which are described below.

\section{Damage of lipid or protein membranes}

The lipid peroxidation induced by paraquat increases in the lung of rodent models and is suppressed by superoxide dismutase; being the hydroperoxide of linoleicoformate acid from the generation of free radicals an important source of cell membrane damage. Paraquat produces selective phosphatidylserine peroxidation of the cell membrane preceding apoptosis in cellular myeloid models.

\section{Excitotoxicity}

It can occur in peripheral organs. It is suggested that NMDA receptors are present in the lungs, and excessive activation may cause an edematous lesion.

\section{Activity related to nitric oxide}

Nitric oxide plays a critical role in tissue injury induced by paraquat, where the toxin peroxynitrite, also charged with cell damage, is produced by reacting with the superoxide radical. Paraquat employs nitric oxide synthase as a source of electrons increasing the concentration of superoxide radicals and paradoxically reducing the amounts of nitric oxide; concluding that the benefits or deleterious effects induced by nitric oxide depend on its concentration.

\section{Ribosilation of ADP}

The effects of oxidative stress induced by paraquat on DNA strands have been demonstrated in porcine models, increasing the activity of ADP ribose polymerase with subsequent reduction of NAD and ATP concentrations. These protein post-translational modifications are activated by lipid peroxidant agents through the activation of cytoplasmic ADP monorribosyl transferases; that later alter the structural conformation of the different proteins that can result in cellular damage.

\section{Apoptosis}

In rodent models, paraquat interferes with the $\mathrm{S}$ phase of the cell cycle of the lung tissue and is related to the peroxidation of the phospholipids of the different cell membranes. Other researchers have reported neuronal cell death in a manner similar to apoptosis related to Parkinson's disease. An experiment with a human lung tissue of 549 cells was incubated for 24 hours with sublethal doses of paraquat, without showing immediate apoptosis characteristics; however, after incubation in a paraquat-free period, signs of cellular apoptosis are recorded depending on time. It was also shown that the use of antioxidants, ascorbic acid, and $\mathrm{N}$ acetyl cysteine, are effective in the reduction of apoptosis induced by paraquat. Although the effect of oxygen on the genesis of apoptosis is unclear, this cellular phenomenon may give way to the production of pulmonary fibrosis.

\section{The function of the mitochondria and multiple organ failure}

Ozawa recognized the importance of the liver in biological homeostasis and considers that a mitochondrial dysfunction plays an important role in the pathogenesis of multiple organ failure. They studied patients with postoperative multiple organ failure by measuring the ratio of ketone bodies (acetoacetate/acid hydroxybutyrate) in arterial blood as indicators of the levels of oxide reduction; indicating that levels lower than 0.4 correlated with severe multiple organ failure and that levels lower than 0.25 are considered lethal. There are two schools that explain the bioenergetic failure in critical patients. One of them affirms that the microvascular damage causes a great variability (in homogeneity) in the capillary flow resulting in hypoxia and in organic dysfunction. The second school states that the bioenergetic failure occurs when there is a decrease in the synthesis of 
ATP consistent with mitochondrial dysfunction; the mechanisms that lead to this condition are the oxidative damage of the mitochondria, the depletion of mitochondrial populations due to autophagy and the inability of the cell to regenerate new mitochondria. Oxidative damage to mitochondria is secondary to the production of reactants from the acute phase, such as superoxides that react with nitric oxide generated by nitric oxide synthase and subsequent formation of peroxynitrite. The latter is a powerful oxidant that directly damages the function of the mitochondrial electron transport complex. In addition, peroxynitrite worsens cellular bioenergetics by activating the ADP ribose polyme-rimerase, which depletes cellular reserves of NAD and limits the mitochondrial synthesis of ATP. The mitochondria subject to oxidative damage, causes apoptosis mediated by increased permeability of the transition pores.

The damaged mitochondria are removed by a process of selfdigestion called autophagy. The mitochondrial loss is compensated with the so-called "Biogenesis" with the formation of new organelles via binary fission. Severe oxidative stress causes an increase in autophagy, reducing the bioenergetic state and limiting the number of functioning mitochondria. A prolonged critical imbalance between autophagy and biogenesis culminates in an irreversible multiple organ failure. A study in septic patients demonstrated an association between overproduction of nitric oxide, antioxidant depletion and mitochondrial dysfunction concluding in multiple organ failure. Clinical recovery from multiple organ failure correlates with increased ATP production.

\section{Mitochondrial DNA injury}

Many polypeptides of the respiratory chain are encoded by the mitochondrial genome which are sensitive to reactive oxygen species, because the mitochondria have a limited arsenal for repair processes. Mitochondrial DNA mutations cause defects in the function of the respiratory chain. In the future, the effects of free radicals induced by paraquat on the mitochondrial DNA of lung tissue must demonstrate the mechanism of pulmonary fibrosis or of a probable chronic poisoning by paraquat.

\section{Transition of the permeability of the calcium dependent inner mitochondrial membrane}

It has been reported that the oxidative damage of free radicals on the mitochondria is triggered by cyclosporin A and the calciumdependent membrane transition, which in turn causes permeability to nitric oxide, depolarization of the membrane, decoupling and cellular edema.

\section{Consumption of NADPH}

Paraquat increases the oxidation of NADPH where its decrease correlates with increased epithelial alveolar damage.

\section{Inhibition of manganese-dependent superoxide dismutase}

Paraquat inhibits the manganese-dependent superoxide dismutase, located in the mitochondria site of greatest injury induced by free radicals. However, superoxide dismutase located in the cytosol(superoxide dismutase dependent on copper and zinc), does not compensate that stored in the mitochondria.

\section{The meaning of lipid peroxidation in paraquat cytotoxicity}

Paraquat decouples oxidative phosphorylation by inducing lipid peroxidation and inhibiting the oxide-reduction chains of ATP synthetase; causing protein damage and cytotoxicity.

\section{The organic multiple failure ${ }^{1-3}$}

Paraquat is characterized by its rapid progression, high mortality and frequent liver and kidney damage. Although it is considered that acute intoxication is asymptomatic in the early stage, our patient enters with multisystemic commitment, due to its time of evolution, and, its geographically remote origin to access a level of care suitable for the management of critical situations. On the other hand, the suicidal intention with a high SAD PERSON score and the refusal of it to be subjected to the initial decontamination measures further overshadow the short and long term prognosis.

In view of initial oxygen desaturation, low PAFI, $\mathrm{PO}_{2}$ less than $60 \mathrm{mmHg}$, and the presence of mucosal scoria lesions, we are forced to protect the airway with orotracheal intubation and management with invasive mechanical ventilation; being aware of the collateral damage that would induce oxygen in the generation of free radicals, as previously mentioned. Grosso modo this lung injury will produce an infiltration of fibroblasts, decreasing the ventilation perfusion ratio; which is exacerbated by the destruction of type I and II pneumocytes, and, thereby altering the alveolar surface tension resulting in subsequent pulmonary fibrosis, evident findings on chest radiography and clinical necropsy.

At the dermal level it has a caustic effect. Ingestion produces irritation in the oral cavity, sialorrhea, ulceration of the upper digestive tract, and mediastinitis. It is common that there is centrilobular intrahepatic necrosis. Shown in Figure 1, mucosal lesions plus icteric dye suggesting liver injury, which is confirmed by the elevation of transaminases, bilirubins, more hepatic congestion evident in pathological macroscopic findings, due to the time of evolution is considered a fulminant hepatic failure with signs of encephalopathy of admission without commitment of coagulation tests or platelet count. Renal tubular necrosis has been reported in $50 \%$ of the cases, with elevated levels of nitric oxide, both of BUN and creatinine with a low glomerular filtration rate, particularly considering that this type of intoxication benefits from renal replacement early, which would begin in the intensive care service of this institution. Finding at the macroscopic level bilateral renal involvement with generalized edema of renal structures. ${ }^{1-3}$ In a study of 68 patients with paraquat poisoning from January 2006 to June 2009, Shi et al., ${ }^{6}$ they registered a mortality of $54 \%$. Of them, the incidence of lung injury was $97.1 \%$ and ARDS was $51.47 \%$. Among the patients who died, the commitment of more than 3 organs was $70.29 \%$, considering in our case lung, kidney and liver injury and the fatal outcome. ${ }^{6}$ Multiple organ failure is an altered immune response to different pathologies of critically ill patients. After the resuscitation of these conditions, the inflammatory response induces distant organ dysfunction, which is not uniform. Culminating in a state of shock, which is defined as an inadequate organic perfusion, where the main affected systems are the respiratory, cardiovascular, renal, hepatic, gastrointestinal, hematological, endocrine and central nervous. ${ }^{5}$ 
The first studies of multiple organ failure were performed in septic patients. However, other causes such as pancreatitis, burns, major surgery, cirrhosis, intoxication, trauma and ischemia-reperfusion syndrome have allowed to unify the pathophysiology of multiple organ failure due to the different hemodynamic, microvascular and oxygen consumption changes. ${ }^{5,7-10}$ Mortality in patients with multiple organ failure is $60 \%$ in patients with dysfunction of 2 white organs, and close to $100 \%$ with the commitment of 4 or more organs 7 .

\section{Altered immune response 1}

The most important pathogenic mechanism in the generation of multiple organ failure is autoimmune dysregulation, where the loss of homeostasis between systemic inflammation and the antiinflammatory response predominates. The innate immune system is a fundamental part of the initial response to different noxas, whose response depends on several factors of the host such as comorbidities, advanced age, inoculum size, and entry portal. In the septic etiology the presence of a microbial infection is detected by mononuclear phagocytes (monocytes, tissue macrophages) and by mast cells. The immune cells contain Toll-Like receptors (TLR) on their surface that allow the host to recognize molecular patterns that are part of the elemental composition of microorganisms; to achieve this, it requires the presence of other molecules such as CD14, MD2 and thus facilitate receptor binding. These receptors activate by nuclear factor kappa beta (nfKB) inside the nucleus; synthesizing multiple pro and anti-inflammatory cytokines. In patients with trauma, the immune system is activated via DAMPs (molecular patterns associated with damage) and there is a mechanism of injury associated with mononuclear phagocytes that trigger the autoimmune response. Injured tissues release proteases that degrade proteoglycans present in the extracellular matrix and the basal lamina of the vasculature. In this process it releases heparan sulfate that acts as an endogenous agonist that blocks the inhibition of the TLR4 and thus promotes the translocation of nfKB. After the innate response, a pro-inflammatory cytokine amplification process continues, such as tumor necrosis factor, Interleukin 1, Interleukin 6, Interleukin 8; being its most important functions the synthesis of other cytokines. The upregulation of tissue factors, vascular cell adhesion molecules type 1 and the synthesis of different enzymes such as neutrophil receptors (L Selectina), endothelial cells (P Selectina, E Selectina ), C reactive protein (which acts as opsonin), nitric oxide synthase, phospholipase A2, and cyclooxygenase. ${ }^{2,5}$ Pro-inflammatory cytokines increase the immune response of neutrophils, which release superoxides and lipid mediators (leukotrienes, prostaglandins), which contribute to the overexpression of adhesion molecules (integrins), and then secrete enzymes, such as elastase, to the vascular endothelium. and the matrix metalloproteinase to be able to effect the diapedesis. This process results in the rupture of the cellular basement membrane and greater capillary leakage. Once the neutrophils have entered the different tissues, they produce a large number of reactive oxygen species, resulting in local oxidative damage. On the other hand, cytokines such as Interleukin 4 and Interleukin 10 would increase an exaggerated antiinflammatory response that would also cause multiple organ failure. The excessive formation of complement C5a plays an important role in the inflammatory response joining mast cells that increase vascular permeability; inducing the expression of p-selectin in endothelial cells; activating the coagulation cascade through tissue factor, and stimulating the formation of proinflammatory cytokines.

\section{Organs injured in multiple organic failure"}

There are variations in the immune response between organs. This phenomenon occurs as a result of the variable activation of the genomic response in the synthesis of pro-inflammatory proteins.

\section{Respiratory system}

Paraquat is a substance that is widely distributed in well-perfused organs such as kidney, liver, heart, and mainly lung because it is a highly vascularized tissue, being affected early regardless of its route of exposure. The average life of paraquat is greater in the lung than in other organs, explaining its greater accumulation, and peak concentration. Thus, animal studies show that the lung is able to accumulate paraquat against a gradient of serum concentration, being the lung concentration 6 to 10 times higher than plasma, explained by an ATP-dependent energy-consuming transport system. The paraquatinduced lung injury culminates in the development of ARDS, which is generally due to the release of proinflammatory mediators such as cytokines, complement, prostaglandins, thromboxanes, leukotrienes; which allows the sequestration of neutrophils in the pulmonary vasculature. During the effector phase, the release of activated leukocytes releases proteolytic enzymes and oxygen species, molecules that mediate lung injury by degrading the proteins of the basement membrane. In ARDS, lung injury can be exacerbated by inducing apoptosis of alveolar epithelial cells with mitochondrial damage, elevation of leukotriene B4 and release of FAS ligand, the latter being a measure of remote organ damage. ${ }^{11,12}$

\section{Two phases of paraquat-induced lung injury are highlighted ${ }^{11,12}$}

a) The destructive phase occurs on the first to third day of exposure, with destruction of alveolar cells type 1 , with cytoplasmic edema, increase in the number of ribosomes and mitochondria with limited exchange of gases through the alveolar membrane. And the alveolar cells type 2 , that present loss of the lamellar bodies with decrease in the amount of surfactant.

b) The proliferative phase: consists of the development of pulmonary fibrosis as a compensatory repair mechanism against the destruction of alveolar epithelial cells with greater synthesis of fibroblasts and greater secretion of collagen, concluding in the limitation of gas exchange and deformity of the normal alveolar architecture. Our patient was in the proliferative phase due to fibrotic findings both at the radiological level and at the pathological level, which determined the irreversible and unfavorable course.

\section{Liver}

Although hepatic perfusion causes acute hepatic dysfunction in patients with circulatory shock, this process resolves after adequate resuscitation. In patients with hepatic failure, an increase in hepatic blood flow as well as increased cytokine production has been demonstrated. The concentration of nor epinephrine seems to induce liver injury by activating adrenergic receptors of Kupffer cells, with higher production of tumor necrosis factor alpha, nitric oxide and IL6. In addition, the presence of elevated levels of Carbamoyl phosphate synthase 1 is an early marker of mitochondrial hepatic injury. ${ }^{11}$

\section{Myocardium and peripheral circulation}

Nitric oxide is produced in high quantities as a consequence 
of the expression of nitric oxide synthetase in the myocardium. Peroxynitrile (formed from nitric oxide and superoxide radicals) causes mitochondrial oxidative damage that causes the reduction of cardiac contractility, and affects oxidative metabolism by activating the polymerase ADP ribose. Elevated levels of nitric oxide decrease cardiac contractility by inhibiting the expression of beta-adrenergic receptors by reducing cytosolic calcium, causing the condition of myocardial hibernation. ${ }^{11}$ As the multiple organ failure progresses, peripheral vasodilation worsens and becomes resistant to exogenous vasoconstrictors. Nitric oxide plays an important role in vasodilation in the early stages, however in late stages cyclic GMP stands out, where erythrocytes stimulate vasodilation in a process dependent on this molecule by synthesizing and storing higher amounts of nitrous oxide that react with the hemoglobin forming the compound hemoglobin S-nitrosothiol.

\section{Brain}

Encephalopathy has been described more frequently in patients with sepsis with a prevalence between 9 and $71 \%$. The aetiology is multifactorial, and there are several theories that suggest its genesis, among them the increase in the permeability of the blood-brain barrier allowing the passage of aromatic amino acids (which act as false neurotransmitters) and inflammatory mediators (cytokines). They emphasize the production of tumor necrosis factor alpha synthesized in microglia and in astrocytes, inducing cerebral edema, neutrophil infiltration and apoptosis. ${ }^{11}$

\section{Digestive system}

There is a unified infectious etiology where the intestine is a potential source of bacteria. The translocation of the same within the systemic circulation is mainly due to decreased intestinal perfusion with mucosal damage and the subsequent barrier mechanisms. ${ }^{5,11}$ In our case, we noticed elevated pancreatic enzymes where, beyond a diagnosis of acute pancreatitis, they can rise in the context of renal failure, however, they can also be correlated with poor prognosis and increased mortality, a finding that was also reported by Li et al. in their 2015 study.

\section{Renal system}

Paraquat is eliminated mainly by the urinary tract, renal failure being oliguria and anuric acid main complication. Proximal renal tubular necrosis is the most relevant histopathological finding, which is due to a lesion induced by free radicals of oxygen that cause lipid peroxidation of the cell membrane. It can also generate a Fanconi syndrome due to glucosuria, phosphaturia, and aminoaciduria. The factors that trigger renal failure are: hypovolemia and sepsis that lead to renal hypo perfusion that results in elevated nitrogen levels and a reduction in the glomerular filtration rate with a reduction in the size of the efferent arteriole. These mentioned factors can trigger the production of inflammatory mediators involved in renal injury, highlighting the tumor necrosis factor and the FAS ligand (inducers of renal cell apoptosis). ${ }^{11,13-20}$ Cheng Chao studied 222 patients intoxicated with paraquat between 2000 and 2012, considering the following items as predictors of kidney injury: Acute hepatitis $(p>$ $0.001)$, time elapsed from the moment of intake until hospital care ( $p$ $<0.001$ ), $\mathrm{PO}_{2}$ low (p: 0.014) and the Paraquat Intoxication Severity Index (SIPP) (0.014), the latter showing the correlation that exists between serum levels of paraquat and the time elapsed since ingestion and its admission to the ICU. found in this particular case except for the execution of the SIPP because our clinical laboratory does not have the capacity to quantify paraquat serum levels.

\section{Hematological system}

The mechanisms that regulate inflammation are similar to those that control the expression of coagulation, where disseminated intravascular coagulation is the final outcome of multiple organ failure. It has been described that platelet counts of less than 80,000 have a sensitivity of $83.3 \%$ and a specificity of $100 \%$ for the prediction of multiple organ failure. Coagulation is initiated through the expression of tissue factor in endothelial cells and monocytes, this process is induced by inflammatory cytokines, integrins or cellular surface components of some batteries such as Bacteroidesfragilis; concluding with the transformation of fibrinogen to fibrin. Although fibrin plays an important role in hemostasis it can also cause intravascular coagulation with limited availability of oxygen in the tissues, the coupling on the thrombin receptor activates nfKB with eventual release of pro-inflammatory factors within which the nitric oxide and the aforementioned tumor necrosis factor alpha, where they also inhibit the transcription and expression of Protein C, paradoxically triggering an effect of coagulopathy of consumption..$^{20,21}$

\section{Discussion}

In the present case we note the failure of three organs on admission to the emergency room, with an APACHE II score of 18 points, SOFA of 11 points, SAPS II of points and MODS 74 points, demonstrating a mortality that ranges between 30 and $88 \%$ basically due to the exaggerated abnormal inflammatory response over the entire body sistems. Although the patient was managed with an organic ventilatory and renal support in a protective manner, the toxicity generated an irreversible systemic damage that ended in the death of the patient.

\section{Conclusion}

Paraquat increases its pulmonary concentration during the first hours post-intake due to its high affinity to alveolar cells. Due to this, injury to the pneumocytes occurs due to the reduction of paraquat to free radical cations (paraquat + ), a process initiated by the NADH. The reactive oxygen species cause a systemic inflammatory response, based on cytokines and cellular infiltration that finally conclude in the peroxidation of the cellular lipid membrane, which in general terms is the basis of generation of multiple organ failure mainly in lung, kidney and liver. The mitochondrial lesion releases iron-free forms, generating greater production of free radicals and systemic apoptosis and necrosis.

\section{Acknowledgements}

None.

\section{Conflict of interest}

The author declares no conflict of interest.

\section{References}

1. Gil HW, Hong JR, Jang SH, et al. Diagnostic and Therapeutic Approach for Acute Paraquat Intoxication. J Korean Med Sci. 2014;29(11):14411449 . 
2. Baltazar T, Dinis Oliveira RJ, Duarte JA, et al. Paraquat research: do recent advances in limiting its toxicity make its use safer? Br J Pharmacol. 2013;168(1):44-45.

3. Seok SJ, Gil HW, Jeong DS, et al. Paraquat intoxication in subjects who attempt suicide: why they chose paraquat. Korean J Intern Med. 2009;24(3):247-251.

4. Fukushima T, Tanaka K, Lim H et al. Mechanism of Cytotoxicity of Paraquat. Environ Health Prev Med. 2002;7(3):89-94.

5. Johnson D, Mayers I. Multiple organ dysfunction syndrome: a narrative review. Canadian Journal of Anesthesia. 2001;48(5):502-509.

6. Shi J, Gao YF, Huang P, et al. Clinical analysis of multiple organ dysfunction syndrome caused by acute paraquat poisoning. Zhonghua Lao Dong Wei Sheng Zhi Ye Bing ZaZhi. 2011;29(7):519-521.

7. Mendonca Pires Ferreira A, Sakr Y, Organ Dysfunction: General Approach, Epidemiology, and Organ Failure Scores. Semin Respir Crit Care Med. 2011;32(5):543-551.

8. Upperman JS, Lacroix J, Curley MAQ, et al. Specific Etiologies Associated With the Multiple Organ Dysfunction Syndrome in Children: Part 1. PediatrCrit Care Med. 2017;18(3):S50-S57.

9. Upperman JS, Bucuvalas JC, Williams FN, et al. Specific Etiologies Associated With the Multiple Organ Dysfunction Syndrome in Children: Part 2. PediatrCrit Care Med. 2017;18(3):S58-S66

10. Møller S, Bendtsen F. Cirrhotic Multiorgan Syndrome. Dig Dis Sci. 2015;60(11):3209-3225.

11. Mizock BA. The Multiple Organ Dysfunction Syndrome. Dis Mon 2009;55:476-526.

12. Dinis Oliveira RJ, Duarte JA, Sanchez Navarro A, et al. Paraquat Poisonings: Mechanisms of Lung Toxicity, Clinical Features, and Treatment. Crit Rev Toxicol. 2008;38(1):13-71.
13. Li Y, Wang M, Gao Y, et al. Abnormal pancreatic enzymes and their prognostic role after acute paraquat poisoning. Sci Rep. 2015;5:17299.

14. Weng $\mathrm{CH}$, Chen $\mathrm{HH}, \mathrm{Hu} \mathrm{CC}$, et al. Predictors of acute kidney injury after paraquat intoxication. Oncotarget. 2017;8(31):51345-51354.

15. Safaei Asl A, Dada shzadeh P. Acute kidney injury in patients with paraquat intoxication; a case report and review of the literature. $J$ Renal Inj Prev. 2016;5(4):203-206.

16. Kim SJ, Gil HW, Yang JO, et al. The clinical features of acute kidney injury in patients with acute paraquat intoxication. Nephrol Dial Transplant. 2009;24(4):1226-1232.

17. Pavan M. Acute kidney injury following paraquat poisoning in India. Iran J Kidney Dis. 2013;7(1):64-66.

18. Kumar H, Singh VB, Meena BL, et al. Paraquat poisoning: a case report. J Clin Diagn Res. 2016;10(2):1-10.

19. Gao Z, Mu DW, Guo L, et al. Etiological factors, prognostic assessment, and outcomes of patients with acute kidney injury and multiple organ dysfunction syndrome. Genetics and Molecular Research. 2014;13(4):8378-8384

20. Marshall JC. Inflammation, coagulopathy, and the pathogenesis of multiple organ dysfunction syndrome. Crit Care Med. 2001; 29(7):S99S106.

21. Miño Bernal JF. Intoxicación por plaguicidas. Montiel Falcon H, Ron Aguirre A, Singer Villalpando A, editors. El ABC de la Toxicología. 1 edición. México: Editorial Alfil; 2017. p . 129-148. 